\title{
Teen smoking cessation
}

\section{R Mermelstein}

Tobacco Control 2003;12(Suppl I):i25-i34

Interest in adolescent smoking cessation has increased dramatically over the past several years, as researchers and practitioners have acknowledged the high rates of adolescents who smoke regularly and the low probability that adolescents who are regular smokers will stop on their own. The evidence base behind smoking cessation interventions for adolescents is also now starting to grow, but unfortunately the studies to date have frequently been plagued by major methodological problems. This paper summarises research conducted on adolescent smoking cessation, notes some of the methodological limitations of prior work, highlights approaches that show promise, discusses some of the challenges involved in addressing adolescent smoking cessation, and makes recommendations for future work.

Correspondence to: Robin Mermelstein, PhD, Deputy Director, Health Research and Policy Centers, $850 \mathrm{~W}$ Jackson Blvd, Suite 400, Chicago IL 60607, USA

robinm@uic.edu
O ver the past several years, interest in smoking cessation for adolescents has mushroomed. This phenomenon reflects a significant change from the tobacco control efforts for youth during the 1970s through the mid 1990s, which had an almost singular concentration on prevention, with little thought or attention paid to cessation. The lack of attention to cessation was based, in part, on several assumptions: (1) that prevention was the more effective means to reduce tobacco use among adolescents; (2) that adolescent smokers were unlikely to be dependent on nicotine and could probably stop smoking if they wanted to; (3) that adolescents were not interested in stopping smoking; and (4) that effective cessation programmes for adults could easily generalise to adolescents. Although one could debate the relative merits of prevention and cessation approaches (with most researchers and practitioners now acknowledging the need for both), research over the past decade has systematically dispelled the second, third, and fourth assumptions. Indeed, it has become increasingly clear that not only is there a need for cessation interventions for adolescents, but there is a demand for them as well. The purpose of this paper is to review briefly the rationale for smoking cessation interventions for adolescents, to discuss the approaches used and outcomes for teen cessation, to highlight the challenges of intervening with adolescent smokers, and to present considerations for future programmes and research. The focus of this review is on cigarette smoking. Although smokeless tobacco use is clearly a problem for adolescents as well ${ }^{1}$ and there are some very promising approaches to smokeless use cessation, ${ }^{2}$ the issues surrounding smokeless use are somewhat different than for cigarette smoking and need to be addressed separately.

\section{THE NEED FOR YOUTH CESSATION INTERVENTIONS}

Rates of frequent adolescent smoking remain unacceptably high. In $2001,19.0 \%$ of 12 th grade students were daily smokers, and $10.3 \%$ smoked at least half a pack of cigarettes a day. ${ }^{3}$ Although these prevalence rates reflect a decline over the past few years, they nevertheless have a long way to go to reach the Healthy People 2010 objective of cutting in half the rate of tobacco use among young people. ${ }^{1}$ Unfortunately, the majority of these adolescent smokers will maintain their smoking well into adulthood. One study estimated that $50 \%$ of adolescent males and females who start smoking as adolescents will continue to smoke for at least 16-20 years. ${ }^{4}$ Although the health consequences of smoking are a function of both the length and amount of smoking, according to a 1994 US Surgeon General report adolescents who are regular smokers are at an increased risk for health problems during their adolescent and young adult years. ${ }^{1}$ Cigarette smoking during adolescence reduces the rate of lung growth, maximum lung function, and overall fitness levels of adolescents, as well as increases the risk of respiratory problems. People who start smoking at younger ages are also more likely to develop high levels of nicotine dependence than are those who start later, leading to more difficulty quitting and accruing more of the negative health effects of smoking. Thus, delaying cessation efforts past the adolescent years has negative health ramifications both during adolescence as well as during the later adult years.

The case for cessation interventions during the adolescent years also can easily be made based on the relatively low rates of "spontaneous" quitting among adolescents. Several longitudinal studies have assessed the prevalence of self initiated cessation among adolescents, and found them to be relatively low. One of the better estimates of self initiated cessation rates comes from a longitudinal study of Australian youth. ${ }^{5}$ Researchers interviewed 937 adolescents at age 15 years, and again at age 18 . At age 18 , only $5.3 \%$ of the adolescents who were daily smokers at age 15 were abstinent for the past month at the time of their interview. Among teens at age 15 who smoked in the last month, but not daily, 33\% had stopped smoking for the year before age 18. Another study with a younger sample (aged 14-16) found a somewhat higher rate of quitting for at least one month (13.6\%). ${ }^{6}$ Using data from the Teenage Attitudes and Practices Survey I (1989) and II (1993), Zhu and colleagues ${ }^{7}$ estimated a

Abbreviations: $\mathrm{BI}$, brief intervention; NOT, Not On Tobacco; TAP, Tobacco Awareness Program; TEG, Tobacco Education Group; YTCC, Youth Tobacco Cessation Collaborative 
$4 \%$ per year quit rate among adolescent smokers who had smoked at least 100 cigarettes in their lifetime and who had smoked at least one cigarette during the past 30 days. Another study ${ }^{8}$ found an overall quit rate (defined as not smoking in the past 30 days) at a one year follow up of $21 \%$ among a sample of 593 adolescent smokers who, at baseline, had smoked any cigarettes during the past month. One of the more methodologically sophisticated studies ${ }^{9}$ of adolescent smoking patterns into adulthood used trajectory analyses to identify different longitudinal patterns of smoking; here the "quitter" group was small, comprising only $6 \%$ of the sample. "Spontaneous" quit rates among adolescents who smoke infrequently are higher, but still surprisingly low. Taken together, these studies suggest that, without intervention, very few adolescent smokers stop smoking.

The spontaneous or unassisted quit rates among adolescents are surprisingly low primarily because of the long held assumption that adolescents "mature out" of smoking or easily quit on their own. However, the rates of spontaneous quitting among adolescents who are regular smokers are not substantially different from those found with adults. Approximately 5-6\% of adult smokers who try to quit smoking at any given time are successful for one month or more. ${ }^{10}$ The similarity in unassisted quit rates between adolescents and adults points to the need for intervention early in the "career" of a smoker.

One reason why adolescents may not readily stop smoking is that they are dependent on nicotine, even before they become regular or daily smokers. The evidence to date suggests that a substantial proportion of adolescent smokers can be classified as nicotine dependent, ${ }^{11}$ with prevalence rates ranging from $19 \%{ }^{12}{ }^{13}$ to as high as $68 \%,{ }^{14}$ depending on the sample characteristics and measurement. Preliminary evidence suggests, too, that nicotine dependence may develop rapidly in a subset of youth. ${ }^{11}{ }^{15}$ For example, DiFranza and colleagues ${ }^{16}$ found that among a relatively young sample (age $12-13$ at baseline) of occasional smokers, $22 \%$ (of 95 subjects) reported a symptom of nicotine dependence within one month of initiating monthly smoking. The majority (63\%) of the occasional smokers in the DiFranza et al study reported one or more symptoms of dependence, and most of these smokers experienced their first symptom before smoking daily. Researchers have also begun to think of nicotine dependence as a multidimensional, dynamic process, ${ }^{11}{ }^{17}$ and that there may be subtypes of nicotine dependence characterised by different configurations of symptoms and different trajectories of acquisition, ${ }^{16}$ such as those who start young and rapidly accelerate or those who have a more slow and steady pace in their escalation patterns. Understanding more about these developmental trajectories of dependence may help improve treatment efforts. Identifying individual differences that influence the pattern and rate of progression across stages of use into dependence may be key to identifying youth who are at high risk for long term smoking and who are most in need of intervention.

Perhaps reflecting their own feelings of dependence on smoking, adolescent smokers frequently report difficulty in quitting or a lack of confidence in their ability to do so. One study found that only a minority (43\%) of a sample of adolescent smokers felt confident that they would ever quit smoking. ${ }^{18}$ Data from the Teenage Attitudes and Practices Survey also show that $74 \%$ of daily smokers reported that it was "really hard to quit". ${ }^{19}$ In contrast is a finding that most of the adolescent smokers they surveyed (72.3\%) felt that they could stop smoking if they wanted to. ${ }^{6}$

Substantial proportions of adolescent smokers do report interest in quitting. In the survey of a large number of Australian students (aged 14-16 years) ${ }^{6}$ about their attitudes toward quitting and prior attempts to quit, most wanted to stop smoking $(64.1 \%)$. In terms of readiness to quit, $29.2 \%$ had not thought about stopping, $42.6 \%$ were currently thinking about quitting, $28.2 \%$ of the weekly smokers were taking action to quit, and $55 \%$ had made a quit attempt in the last year. Interestingly, more females than males were taking action to quit. Another study ${ }^{20}$ examined readiness to quit in a large longitudinal sample of adolescents who were surveyed three years apart (average ages 14.4 and 17.4). Only $11.6 \%$ of the smokers had quit over the three year period, about $19 \%$ were seriously considering quitting within the next six months, and 32\% reported that they had not thought about quitting, even when they were older.

Researchers have also examined stages of the cessation continuum, as laid out by the transtheoretical model of change (stages of change), in a sample of high school students. ${ }^{21}$ They identified six cessation stages: (1) recent acquisition $(5.9 \%$ of the sample; those who had been smoking less than six months, regardless of their intentions about quitting); (2) precontemplation $(35.4 \%$ of the sample; smokers not thinking about quitting in the next six months); (3) contemplation $(30.7 \%$; smokers thinking about quitting in the next six months, or those thinking about quitting in the next 30 days, but who had never had a serious quit attempt before); (4) preparation (14.6\%; smokers thinking about quitting in the next 30 days and who had at least one serious quit attempt within the last six months); (5) action (4.7\%; former smokers who had quit smoking in the past six months); and (6) maintenance $(8.7 \%$; former smokers who had been abstinent more than six months). Such studies ${ }^{20}$ provide $^{21}$ important data about the readiness of the adolescent smoking population for a quit attempt; at any given time, about 15-19\% of adolescent smokers are seriously considering quitting in the near future, thus providing a good target for intervention, and an additional 30\% could become "primed" for future intervention efforts.

Adolescents' reasons for wanting to stop smoking also may provide intervention planners with key motivational hooks. Across several surveys, ${ }^{22} 23$ health concerns are listed as the most frequently cited reasons that adolescents want to quit. A sample of somewhat older adolescents (16-20 years) ${ }^{24}$ drawn from vocational colleges and unemployment settings found that saving money was the reason most cited $(57 \%)$ as a motivator to convince this population not to smoke. In addition, becoming a parent, going out with a non-smoker, and seeing someone ill from smoking were frequently endorsed as motivators. Daily smokers may be more likely than occasional smokers to report wanting to quit because they are "addicted". ${ }^{23}$ Other less frequently cited motivators have included social pressure and a feeling that smoking presents an unacceptably bad image.22

Although adolescents may endorse wanting to quit, these sentiments may still be somewhat abstract to them; they may not necessarily be mobilised to do something about quitting, or even to know what to do. Balch explored adolescents' thoughts and knowledge about quitting in a qualitative focus group study. ${ }^{25}$ One of the more important findings to emerge from the focus groups was the adolescents' hesitancy about how they would go about quitting and their lack of ability to formulate a concrete plan or to know where to go for help. Stanton's quantitative survey ${ }^{22}$ similarly found that the vague "use of willpower" was the most frequently cited method adolescents had tried for quitting, but that it also was one of the most frequently cited as not helpful. In that study, only 3\% of the adolescents mentioned trying a recognised programme or method to help them to quit.

In summary, when one considers the prevalence of adolescent smoking, the negative health consequences of smoking both during the adolescent years and the cumulative damage over time, the relatively low rates of youth quitting on their own or "maturing out" of smoking, and adolescents' interest in stopping smoking, the need for cessation programming during the adolescent years is clear. 


\section{OVERVIEW OF INTERVENTION APPROACHES WITH ADOLESCENTS}

Smoking cessation interventions for adolescents are still in their relative infancy, and published empirical studies number less than 50. Many of these studies, however, are plagued with methodological problems, including poorly described interventions and methods, inadequate measures of cessation (for example, self report as "being quit" without any behavioural referents), brief follow ups, poor retention rates, and lack of control or comparison groups. Sussman recently reviewed 66 cessation reports as an update ${ }^{26}$ to his 1999 review of the field. ${ }^{27}$. He covered all organised programmatic efforts to reduce youth smoking. Programmes reviewed included both school based and health care based clinics, classroom based interventions, computer expert systems, family programmes, policy efforts, mass media programming, and multicomponent statewide programmes. Only 47 of the 66 studies $^{26}$ were published in peer reviewed journals, and no studies were excluded because of poor quality. Fifteen of the 66 studies were randomised experimental trials, and 22 used a quasi-experimental design, in which treatment groups may have been matched or in which control groups were chosen to compare natural cessation rates. The remainder of the studies $(\mathrm{n}=29)$ utilised a single group design without a comparison or control group.

The Sussman review is noteworthy for its comprehensive identification of programmes, its detailed examination of treatment effects by theoretical approaches, modality or channel of intervention, and potential moderators of intervention effectiveness (for example, sex). Rather than reiterate the detailed listing of the results by study, this paper will cover some of the highlights of the Sussman review, comment on some of the conclusions, and then consider some exemplary cessation approaches in more detail.

The theoretical constructs behind the cessation programmes offered to date have varied greatly. Sussman identified eight theoretical frameworks used across the 66 studies. Broadly, these included: (1) social influence models, such as teaching ways to combat social influences or perceptions that promote tobacco use; (2) cognitive-behavioural approaches, which tend to emphasise self management and skills training; (3) motivational enhancement, in which the emphasis is placed on clarifying ambivalent feelings about quitting and highlighting positive expectancies about cessation; (4) response-contingent reinforcement, where incentives are given for behaviour change; (5) supply reduction approaches, which tend to be more typical of policy oriented programmes and are usually either price increases or restricting access to tobacco; (6) addiction focused approaches, which include pharmacological approaches or other ways of coping with withdrawal; (7) transtheoretical model of change (stages of change) approaches, in which interventions may be tailored to an individual's level of readiness to change; and (8) affect clarification, which emphasises techniques meant to clarify conflicting feelings and moods. Although many studies have focused on only one of these approaches, others have drawn from several models simultaneously, such as combining social influences and cognitive behavioural approaches ${ }^{28}$ or motivational enhancement with stage based assessment and tailoring. ${ }^{29}$ As will be discussed later, there is little direct research to date directly comparing these approaches.

Cessation interventions also have been delivered in a variety of settings or modalities. School based, multi-session, group programmes have been the most commonly used approach, ${ }^{28}$ but also tend to have a limited reach; students must be "ready for action" at the point of programme offering. Health care delivery settings have also been used for delivering cessation interventions, including the use of motivational interviewing techniques in emergency room visits ${ }^{30}$ or school based health clinics. ${ }^{29}$ Internet based, virtual "chat room" formats have also been tried as a means of potentially increasing the geographic reach of more traditional group programmes. ${ }^{31}$

As Sussman notes, ${ }^{26}$ there are enormous methodological challenges in drawing conclusions across these studies. For example, inclusion criteria vary greatly, with some studies including total populations ${ }^{32}$ and thus having the full range of smoking levels, and others including only daily smokers who smoke at least a given number of cigarettes a day. ${ }^{14}$ Also problematic are the varying definitions of cessation, which have ranged from adolescents' self definition of "being quit" or a "former smoker" ${ }^{\prime 33} 34$ to biochemically validated abstinence. ${ }^{14} 30$ Criteria for cessation also have varied by whether researchers choose to report data on cessation rates only for participants who completed an intervention or for all those assigned to an intervention condition (an intent-to-treat analysis). Sussman calculated a mean retention rate of $78 \%$ across the studies that provided attrition data (only 39 of 66 studies). ${ }^{26}$ The follow up periods for the interventions also have varied considerably, although Sussman reports that the modal length of follow up was six months. Considering the notoriously high relapse rates for smoking cessation found in the adult literature, ${ }^{35}$ one needs to be very cautious about drawing conclusions from cessation interventions from studies with follow ups of less than six months.

Sussman calculated average quit rates across the studies, and found an immediate post-programme quit rate of approximately $14 \%$ for the intervention groups, compared to approximately $7 \%$ for control conditions. ${ }^{26}$ Similarly, he found that quit rates at follow up dropped slightly to $12 \%$, but were still almost double those found for control groups (7\%). The quit rates for these control groups fall within the range of those found for spontaneous quitting among adolescents noted earlier. The positive conclusion drawn from his review is that teen cessation programmes can produce higher quit rates (almost double) than those of control groups. In part, this conclusion is based less on a formal analysis of methodologically strong studies than it is on an accumulation of data, regardless of quality, across many studies, showing trends for better outcomes for intervention conditions. Nevertheless, it provides encouragement for a fledgling field.

Because of the methodological challenges of comparing data across the assortment of youth cessation studies to date, and difficulties in completing a more formal meta-analytic review of the cessation field, the Youth Tobacco Cessation Collaborative (YTCC) comprised of representatives from major organisations with interests in youth tobacco cessation (American Cancer Society, American Legacy Foundation, American Lung Association, Canadian Tobacco Control Research Initiative, National Cancer Institute, National Cancer Institute of Canada, National Heart, Lung and Blood Institute, National Institute on Drug Abuse, and the Robert Wood Johnson Foundation) used a group review process to validate the findings of Sussman's review. The review panel was comprised of members of the YTCC and researchers in the area of youth tobacco cessation. The goal of their review was to create a document identifying "better practices" for the field and serve as a guideline for making decisions about how to help youth stop smoking. Although the initial hope of the YTCC group was to identify "best" practices, the group consensus was that the data are currently lacking for such designations. (The YTCC evidence review group first rated studies on internal validity and overall quality, based on a variety of methodological criteria, including design, sample size, follow up time, adherence to intervention, retention, and other criteria. The full summary of their findings will be published shortly, and were discussed during the annual meeting of the Society for Research on Nicotine and Tobacco, New Orleans, February 2003. In contrast to the overall optimistic 
conclusions of the Sussman review, the YTCC evidence review group drew more guarded conclusions, identifying approaches that held promise (for example, cognitivebehavioural approaches), approaches for which inconclusive evidence exists (for example, pharmacological approaches; largely due to limited numbers of studies and designs), and approaches that would not be recommended because of potential for harm or based on expert opinion (for example, fear appeal alone or sensory deprivation). The YTCC guide will provide an important document to help programme developers and practitioners make decisions about interventions to help youth stop smoking.

\section{SPECIFIC CESSATION APPROACHES AND OUTCOMES}

Described below are examples of studies of specific cessation approaches with teens. The goal here is not to be comprehensive (see Sussman ${ }^{26}$ for a complete listing of studies to date), but rather to provide illustrations of the main approaches that have been used with adolescent smokers along with their relative success rates. Unfortunately, with the studies conducted to date, it can be difficult to tease apart specific approaches (for example, cognitive-behavioural skills training, motivational interviewing) from the channel of delivery (for example, school based clinic, health care setting, internet). It may well be that both approach and delivery setting affect outcomes. For example, the delivery channel itself may increase the potency of motivational messages (such as those delivered by a respected health care professional versus unfamiliar volunteer) or may decrease the effectiveness of specific approaches (such as trying to teach skills training within limited time constraints). Until more studies are conducted from which one can separate the content or substantive approach from setting or delivery channel, the review of approaches below unfortunately maintains this confound.

\section{School based clinics}

The most widely used intervention setting across studies has been school based clinics, in which tobacco cessation programmes are delivered to small groups of students at school. ${ }^{28336}$ These group sessions are not a part of any regular classroom curriculum, but are devoted specifically to addressing cessation. The sessions could be held either during regular school hours, in which case the student must have permission to miss scheduled classes, or after school. Turner $\mathrm{et}_{\mathrm{al}} \mathrm{l}^{37}$ evaluated school based clinics at 29 schools in Illinois and found that sessions scheduled during school hours had higher recruitment and retention rates than those scheduled after school. Although most participants in these programmes are volunteers, others may be mandated to attend the cessation clinics, often in lieu of alternative punishment or school suspension for being caught smoking.

One of the more promising school based clinic approaches is the American Lung Association's Not On Tobacco programme (NOT). As described by Dino and colleagues, ${ }^{28}{ }^{38}$ the NOT programme is comprised of 10 weekly, 50 minute group sessions, conducted during school hours, delivered in same sex groups by trained facilitators, who also are sex matched to the group membership. Topics covered in the group sessions include motivational issues, smoking history, consequences of smoking, preparation for quitting, dealing with urges and cravings, stress management, dealing with family and peer pressures, increasing healthy lifestyle behaviours, and relapse prevention.

Dino and colleagues used a matched two group design to compare the NOT programme with a brief intervention (BI) condition. The BI programme was also offered as a group based programme during school hours, but with mixed sex groups. In the BI condition, students received 5-10 minutes of scripted quit smoking advice and self help brochures. Quit rate was determined by a combination of self reported quitting and a validated expired air carbon monoxide reading. Only students who reported smoking five or more cigarettes a day were included in the analyses. At the follow up (approximately 5.2 months post-programme), only $50 \%$ of the original sample of 566 participants provided data. The carbon monoxide validated quit rates were $21.7 \%$ for the NOT participants compared to $12.6 \%$ for those in the BI condition. However, the intervention effect was limited only to females. For males, there was no difference in quit rates between the two conditions ( $14.4 \%$ quit in NOT and $15.9 \%$ quit in BI). For females, though, the NOT programme produced quit rates more than three times greater than the BI condition $(29.6 \% v$ $8.9 \%)$.

As Dino and colleagues note, one of the important points to consider in their evaluation of the NOT programme is the relatively high rate of cessation in the BI condition, and the potentially unique environmental conditions occurring at the time of the evaluation. Their evaluation was conducted in Florida at a time when there was considerable attention paid at the state level to anti-smoking efforts, and the overall climate may have enhanced both recruitment and cessation rates for both conditions. This evaluation of the NOT programme is noteworthy for its relatively large sample size and use of biochemical validation. Limitations of the study include the use of a matched design, substantial dropout at follow up, and lack of clear behavioural referents for defining cessation. However, the results for females are nevertheless very encouraging. What is not clear, though, is why males failed to benefit from the NOT programme. More data on process variables and predictors, broken down by sex, would be useful in trying to explain further this sex effect. Future evaluations of NOT also should consider whether the format of same sex groups (versus mixed sex groups) is necessary or even beneficial.

In one of the better designed and evaluated studies of school based clinics, Sussman $e^{2} a^{36}$ developed a smoking cessation programme for continuation high school students and compared the basic programme to one enhanced by a "school as community" component. Continuation school students report substantially higher levels of cigarette smoking than do traditional high school students. One of the strengths of this programme-"Project EX"-was the use of input from continuation school students in developing the programme activities and modality of delivery. The final clinic programme consisted of eight group sessions delivered over a six week period. Session topics focused on discussion of reasons for smoking and quitting, dealing with family and friends, healthy ways of coping with stress, understanding the dangers of tobacco, making a commitment to quit, discussing withdrawal and ways to manage it, avoiding false expectations, practising yoga to maintain a sense of balance in one's life, learning maintenance strategies such as anger management and relaxation, and avoiding relapse. Topics and activities were presented in engaging formats (for example, games, "talk shows").

Sussman et al randomised 18 schools to one of three conditions: (1) the clinic programme only; (2) the clinic programme plus a school-as-community component; or (3) a standard care control. ${ }^{36}$ The school-as-community component included efforts to enhance anti-tobacco messages in various formats throughout the school community, in an attempt to promote attitude shifts and increase motivation to quit. A notable feature of this study was its excellent recruitment rate: $34 \%$ of the target population of smokers enrolled in the programme $(\mathrm{n}=335)$. The vast majority of students $(85 \%)$ enrolled were daily smokers with $75 \%$ scoring in the moderate to high range on measures of dependence. Follow up, with $51 \%$ of the participants responding, occurred an average of 3.7 months after the last clinic session. The primary outcome measure was 
30 day abstinence at follow up. No differences were found between the two active programme conditions. Among those who completed the programme (54\% of those enrolled), $30 \%$ were abstinent at follow up compared to $16 \%$ for the controls. Using an intent-to-treat analysis, assuming that those lost to follow up were smoking, the quit rate fell to $19 \%$ for the programme conditions, compared to $10 \%$ for the controls, still a statistically significant difference. Smokers who were most likely to quit were those who scored lower on measures of dependence.

There are several strengths to this study: the programming was developed in conjunction with the target population and was well rated by participants; the design of the evaluation was a randomised trial; and the quit rates were substantially better for the programme conditions than for the controls. Although the attrition rate was substantial for the programme (46\% of those enrolled did not complete the programme), higher attrition rates may be expected with this population. In addition, even with the intent to treat analysis, the condition difference remained significant. This study is also notable for its targeting of a very high risk group.

Taken together, both the Dino et al studies ${ }^{28}{ }^{38}$ and Sussman et $a l^{36}$ study provide good support for the potential efficacy of school based group programmes that are developed in consultation with the target audience of adolescents, have programming that is fun and enjoyable to the youth, and follow principles based on cognitive-behavioural approaches, with an emphasis on coping skills training and alternative ways of handling situations or negative moods without smoking. An important point to note, too, is that these studies were conducted with adolescents who participated in the cessation programmes voluntarily, who were not "mandated" to participate, and who were regular smokers. These studies also highlight, though, the substantial problem of dropout both during the programme and with follow up evaluations.

In contrast, Coleman-Wallace and colleagues ${ }^{33}$ describe the evaluation of two complementary, school based programmes (the Tobacco Education Group and the Tobacco Awareness Program) developed to address the needs of adolescent tobacco users at different stages of readiness to change-not just those volunteering and ready to quit. The Tobacco Education Group (TEG) was designed to increase a smoker's motivation to quit and includes discussions of personal reasons for smoking, pressures to use tobacco, consequences of tobacco use, and demonstrations of short term consequences of use. The Tobacco Awareness Program (TAP) was designed as a cessation programme for adolescents who are ready to quit. Both programmes have eight one hour group sessions. Coleman-Wallace et al report results of implementing TEG and TAP in six Southern California high schools. Participants in the TEG programme were 201 adolescents who were mandated to attend the programme in lieu of suspension for smoking. Participants in the TAP programme comprised 101 volunteers and 27 students mandated to attend (despite being designed for those who were ready to quit). Control participants $(\mathrm{n}=22$ students $)$ did not receive any programme, and were recruited separately. No random assignment was used in the evaluation. At the end of the programme, $12 \%$ of the TEG participants and $15 \%$ of the TAP participants reported quitting. No behavioural definitions of quitting were provided. Interestingly, those mandated to attend TAP (the programme designed for those ready to quit) had a lower quit rate $(9 \%)$ than those who volunteered (16\%). Although the TEG programme was designed to follow a "stages of change" model and to move students who had little interest in quitting to start to consider or to take action toward quitting, TEG did not decrease the prevalence of precontemplation. There were several limitations to this study, including the lack of random assignment, the very small control group (which essentially restricted its utility), and the lack of any criteria for "quitting". The results for TEG were particularly disappointing in that little movement was seen in readiness to consider quitting, which was its primary aim. However, this study is useful in considering the implications of mandating youth to attend formal programmes, whether for motivation enhancement or for quitting. The results of this evaluation suggest caution in the use of recommending cessation programming in lieu of school suspension, and call for a need to further study potential unintended consequences of such policies.

\section{Pharmacological approaches}

Two studie ${ }^{14}{ }^{39}$ have utilised nicotine patch therapy with adolescent smokers who reported relatively high rates of daily smoking (20 cigarettes per day or more). In both studies, smoking cessation counselling was also provided. Although the patch was well tolerated and safe among the adolescents, the overall quit rates were substantially lower than those found with adults (for example, $14 \%$ at end of treatment and $4.5 \%$ at six months in one study, ${ }^{14}$ and $5.0 \%$ at six months in the other ${ }^{39}$ ). In contrast, the estimated abstinence rates for adults using the nicotine patch from meta-analyses of 27 studies is $17.7 \% .^{40}$ These studies are clearly disappointing in terms of their overall success rates, but they also call for more placebo controlled studies of pharmacological approaches with adolescents. Considering that these participants had higher baseline smoking rates than those of participants found in most other adolescent smoking cessation studies, we need to have a better sense of how youth with higher levels of dependence would do with comparative approaches.

\section{Interventions delivered in health care settings}

Interventions delivered in health care settings have the appeal of broad reach and the backing of findings from the adult literature of significant improvements in quit rates among smokers who receive brief provider delivered interventions. In addition, several professional organisations have developed guidelines and recommendations from expert consensus panels for addressing tobacco use in adolescent patients' health care visits (for example, American Academy of Pediatrics, American Medical Association). However, the few studies that have examined the effect of brief interventions in healthcare settings have had mixed results. ${ }^{41}{ }^{42}$ One promising study by Colby and colleagues ${ }^{30}$ identified smokers during hospital visits to the emergency room, outpatient clinics, or inpatient unit. Smokers were then randomly assigned to receive either brief advice or a motivational interview. At a three month follow up, $20 \%$ of the motivational interviewing group were abstinent, compared to only $10 \%$ of those in the brief intervention group. Although these abstinence rates were not statistically significant with the small study sample size, they nevertheless show promise for this approach. This study is notable for its strong methodological features, including a randomised design and biochemical verification of abstinence.

A more recent, ongoing study of a cessation intervention offered in the context of routine medical care also offers encouraging preliminary results. Hollis and colleagues ${ }^{43}$ conducted a randomised trial of brief clinician advice, the Pathways to Change interactive computer program, and brief motivational counselling to reduce smoking among 14-17 year old smokers seen at primary care visits. This intervention was population based and individually tailored, and it took advantage of both the teachable moment in a medical visit as well as the attractiveness of computers to teens. Teens were randomly assigned to receive either a tobacco intervention or brief dietary advice. Among regular smokers at baseline, 23\% of the tobacco group compared with $13 \%$ of the control group were abstinent (defined as not smoking in the past 30 days) at one year, and a significant difference between conditions was also maintained at a two year follow up. These rates are impressive in absolute terms ( $23 \%$ abstinent at one year), and 
perhaps more so when one considers that this was a population based approach to cessation. This study also highlights the potential of combining modalities of approaches (for example, brief motivational counselling and interactive computer programming).

\section{Internet approaches}

The internet presents an appealing avenue for future cessation interventions. The promise of the internet as a vehicle for delivering interventions to teens is great: a large proportion of teens have access to the internet; there is the potential to tailor information at an individual level; it provides relative privacy or anonymity that may be important to teens; and the possible impact, as a function of its reach and relative cost effectiveness, is great. In addition, the internet has the potential to create social support networks of teen quitters, through chat rooms and similar vehicles, which may facilitate initial cessation and enhance long term abstinence.

Cheh et al recently evaluated smoking cessation internet sites on informational content, usability, source credibility, and the currency of information presented..$^{44}$ They restricted their review to sites that provided information in English and that were free to the public. They reviewed 30 sites, most of them oriented not directly to teens, but rather to adult smokers. Most provided accurate information, but were rated less highly on accessibility, credibility, and currency. Also, reading level was relatively high (slightly more than 50\% at higher than grade 8 level), which could present problems for many teenagers.

Websites that seem to be more geared to youth tend to have more "flash", animation, feature stories about teens who are trying to stop smoking, contests, and teen oriented clubs and chat rooms. In addition, on these sites, quitting tips consider reasons for smoking and strategies that are reflective of teen issues (for example, feelings of rebellion, wanting to appear older, breaking up with friends, peer and family issues). Some of these sites can be found within the broader framework of teen health sites, and others are smoking specific.

Unfortunately, there have not yet been any formal evaluations of the efficacy or use of internet websites for teens. Several ongoing studies are exploring the use of websites as components or adjuncts to other approaches (for example, in conjunction with brief provider advice or as an adjunct to a group based programme), but no preliminary data are yet available.

\section{Other approaches}

Two other modalities for intervening with adolescents that have intuitive appeal are family based interventions ${ }^{45}$ and computer assisted approaches. ${ }^{21}$ Interventions involving families are appealing because of the potential to take advantage of naturally occurring support structures and because the family remains one of the most important social contexts and influences for adolescents. Similarly, computer based approaches have appeal for youth because of their potential for individual tailoring and familiarity of use among adolescents. Both of these approaches also have the potential for broad reach. Unfortunately, the data for both are only suggestive, with no significant differences found between intervention and control in the family based study ${ }^{45}$ (although the difference was in a promising direction), and with no condition differences in the computer assisted study as well. ${ }^{21}$

\section{Summary of specific approaches}

The above examples demonstrate the range of approaches tried to date with adolescent smokers. When examined on an individual level, the results are somewhat discouraging. But they represent what may be considered a "first generation" of studies with adolescent smokers, and as such, suffer from many of the methodological difficulties that are common in early studies in many fields. As researchers learn from these important first line approaches, we may expect improvements in both design and outcomes.

\section{CHALLENGES OF INTERVENING WITH ADOLESCENT SMOKERS: WHY ARE SUCCESS RATES SO LOW?}

Practitioners and researchers in the field of adolescent smoking cessation readily acknowledge the difficulties both of mounting interventions with this population and of achieving reasonable success rates. The challenges of intervening with adolescent smokers come from multiple frontscharacteristics of the individual adolescent, the behaviour of smoking itself, and the macro-environment surrounding adolescent smoking.

In considering the challenges of adolescent smoking cessation, it may be useful to identify steps leading to successful cessation and the barriers that need to be addressed at each point in the behaviour change process. At a very fundamental level, adolescents who smoke, even occasionally, need to acknowledge their behaviour, self identify as a "smoker," and realise that messages or interventions for cessation apply to them. However, patterns of occasional smoking among teens may make it less likely for a teen to self identify as a "smoker" and as someone who needs to change. Indeed, qualitative work from focus groups with adolescents suggest that teens perceive a difference between being a "social smoker" (often defined optimistically by teens as having "control" or "choice" over one's smoking) or a "real smoker" (someone who is "addicted"). ${ }^{46}$ Lack of identity as a "smoker" may lead teens to dismiss cessation messages as not applicable to them.

Motivating adolescents to stop smoking and mobilising them to take action also present challenges. As noted earlier, although adolescents may report that they want to quit, specific plans for quitting often are relatively vague and far in the future, and adolescents may lack the knowledge for how to go about quitting or where to seek help. Also, there is the potential issue that smoking is a "prohibited" and often punishable behaviour for adolescents. Thus, adolescents may be reluctant to seek help for quitting if doing so means that they may suffer negative consequences for admitting to smoking. Programme developers need to work particularly hard on ensuring confidentiality for youth who seek help. In addition, careful consideration needs to be given to the issue of requiring parental consent for interventions. Most behavioural interventions pose minimal risk to the adolescents, and programme developers, researchers, and institutional review boards should weigh the pros and cons of requiring parental permission if obtaining such permission reduces the likelihood of an adolescent seeking treatment. ${ }^{47}$

Smoking cessation interventions also must fit seamlessly into the broader tobacco control environment as perceived by adolescents. From a universal intervention perspective of reducing tobacco use among adolescents, it can be difficult to balance messages and policies for both prevention and cessation. On one hand, we know that strong enforcement of antitobacco policies discourages use, and communication of these policies and consequences is important for reducing use. On the other hand, we need to consider how these messages may be interpreted by adolescent smokers who are motivated to quit (perhaps in part as a result of such messages) but who may also be reluctant to come forward because of strong enforcement policies. Even if immunity from punishment is granted to smokers who seek help in quitting, we need to be careful about how to balance the multiple messages and their interpretations by youth. More work needs to be done in understanding how the broader tobacco control environmental messages are interpreted by adolescents at all stages of the uptake and cessation continuum. For example, does heavily promoting the availability of smoking cessation interventions 
for teens give others the false impression that smoking is more prevalent than it is? Or do these messages perhaps give the mistaken impression that help with quitting is readily available, quitting is easy, and that interventions are very successful? Understanding more about how adolescents respond to varying anti-tobacco and smoking cessation messages may help us to craft more effective recruitment strategies, and perhaps to establish norms supporting cessation.

Once adolescents are motivated to stop smoking and mobilised to take action, we need to have developmentally appropriate interventions available and accessible to them. Adolescence is a time when making any behaviour change may be difficult. It is a developmental stage characterised by a series of changes in all domains-physical, emotional, cognitive, and social. One noted challenge ${ }^{48}$ is that adolescents must move from parental monitoring, control, or support of health related behaviours to more self regulation. However, self regulation skills may not be well developed yet. For example, the cognitive skills required for successful behaviour change include the ability to identify and self monitor behavioural patterns, anticipate problem situations, develop plans for handling difficult times, and then remember both the plans and the need to take action in the future. These cognitive skills are not well developed in many adolescents, and intervention programmes may need to be more sensitive to the cognitive developmental variation among adolescents than they are currently. Simply taking strategies and presentations that are developed for adults and putting them into the jargon of adolescents or imbedding them in fun formats does not necessarily overcome the cognitive complexities of the strategies involved. Importantly, the association between attention deficit disorders and smoking ${ }^{49}$ among adolescents may further exacerbate the cognitive challenges of behaviour change. What this may mean is that interventions need to provide more structure and support over longer periods of time than is typically the case with adults.

Similarly, emotional factors and changes in emotionality during adolescence may contribute to adolescents' difficulty in quitting. Not only is smoking associated with a variety of emotional problems in adolescents ( such as depression ${ }^{49}$ ), but adolescents also report smoking to help manage mood ${ }^{46}$ Considering the increased fluctuations in mood during adolescence, ${ }^{48}$ stopping smoking may be particularly difficult at this time. Interventions that incorporate mood management skills geared towards adolescents may be needed for some youth.

The issue of personal control presents another challenge. Personal control is at the core of many behaviour change efforts, and adolescents, compared to adults, have less control over their lives, environments, and potential reinforcers for change. The challenge for interventions is to develop strategies that are available and within adolescents' means to access and to use.

A challenge, too, in helping adolescents to stop smoking rests with the behaviour of smoking itself. Not only are many adolescents dependent on nicotine ${ }^{11}$ and thus likely to experience unpleasant withdrawal symptoms when they quit, but their patterns of smoking may be less predictable and more opportunistic than those of adults, and therefore harder to anticipate, plan for alternatives, and to change. For example, compared to adults, adolescents are more likely to smoke more irregularly and to smoke less per day than adults. ${ }^{50}$ These intermittent patterns may also become very reinforcing in themselves.

There is also the challenge of maintaining abstinence once initial quitting is achieved. Transitions are a hallmark of adolescence, including transitions in schools, living arrangements, social connections, work, and the transition of becoming a non-smoker. Life event transitions may be both opportunities and risks for the maintenance of abstinence, and interventions need to be extended to consider the maintenance and relapse processes as well during adolescence.
Finally, it is important to understand that the considerable changes that occur throughout the adolescent years have implications for whether we really can have a "one size fits all" intervention for all of the teen years, or whether we need to consider developmentally based subgroup interventions. Interventions that are appropriate for 14 or 15 year olds may not be ideal for 17 or 18 year olds. Given the complexities of the period, it may be important for us to consider a sequence of developmentally appropriate strategies for each year or grade, and also to consider the need for long term or multiyear interventions, knowing that strategies that might have worked for a high school freshman may now fail that ex-smoker as a high school senior. Interventions that plan for developmental and life changes may have more long term success.

\section{DISCUSSION AND RECOMMENDATIONS FOR FUTURE WORK}

This is an exciting time for the field of adolescent smoking cessation. There is strong demand for successful cessation interventions from many stakeholders-adolescents, parents, schools, the health care delivery system, and importantly, the public health community who want to spend available funds for tobacco control wisely. Given the increasing attention to the problem of adolescent smoking over the past decade, there is a diverse and receptive audience for adolescent smoking cessation programmes.

In drawing conclusions about the efficacy of smoking cessation interventions for adolescents, we need to address two primary questions. First, have youth smoking cessation interventions been tested adequately, and second, do they produce higher abstinence rates than those found with control groups? Considering the multiple methodological weaknesses in many of the published reports to date addressing adolescent smoking cessation, one could easily argue that many of the smoking cessation approaches geared towards adolescents have not been rigorously evaluated. As the above review suggests, there are no unequivocal successes that have met the standards of high quality, replicated studies. There are promising approaches, however, that can be delivered in a variety of settings, ranging from clinic based programmes in school settings ${ }^{283638}$ to more minimal interventions delivered within health care settings. ${ }^{30}$ There are also several well designed studies, funded by the National Cancer Institute and others, that are currently in the field and will contribute substantially to our knowledge base over the next few years. Some of these studies examine the effectiveness of interventions within health care delivery systems, others with internet or interactive computerised interventions, and others with special populations (for example, youth with co-morbidities) or pharmacological approaches. Thus, we are likely to see a continued growth of better designed studies, with hopefully continually improving outcomes, over the next several years.

We also are not yet at a point where we can make reasonable statements about the relative efficacy of varying approaches. Given the differences in study samples, measures of cessation, and lengths of follow up, it is almost impossible to find a common metric from which one can compare study results. Recommendations about methodological issues in adolescent smoking cessation studies are now available to researchers, though. $^{50}$ These recommendations were developed with the hope that they would enhance the likelihood of conducting comparative analyses across studies in the future. These recommendations include encouraging researchers to use multiple measures of baseline smoking rates and patterns; to clearly report inclusion criteria, recruitment methods, recruitment rates, and retention data; to include adolescents' self perceptions of their smoking status, labels, and intentions; to use a variety of outcome measures that go beyond point prevalence, such as considering intermediate outcomes (for 
example, quit attempts, numbers of consecutive days abstinent); to use a 30 day criterion for abstinence; to consider options for verification of self report; to have multiple time points for follow up; and to report relapse rates.

Do smoking cessation interventions for adolescents produce higher quit rates than those obtained with control groups? There are several difficulties in answering this question. A key question for the field to address is what should be the appropriate benchmark for success for adolescent smoking cessation programmes. Should they be better than "spontaneous" quit rates, or should they be better than controls? Should they be at least as good as those found with adults? The majority of published studies of smoking cessation interventions with adolescents do not have appropriate randomised control or comparison groups. Given the difficulties in obtaining good estimates of "spontaneous" quit rates, one should question the validity of using such a comparison. In addition, considering that the "standard of care" for adult smokers is, at a minimum, brief advice to stop smoking, one would be hard pressed to argue that we should accept less than that as a minimum comparison group for adolescent smoking cessation interventions. Despite these cautions, there is reason to be optimistic about the effectiveness of youth smoking cessation interventions. As Sussman ${ }^{26}$ has concluded, broadly speaking, there may be an accumulation of evidence suggesting that quit rates for interventions are better than those found for controls. In terms of specific approaches, the evidence may be most promising for interventions that follow cognitivebehavioural principles of change, which include self management training, coping skills training, problem solving, and specific techniques for enhancing motivation through dealing with withdrawal. In terms of delivery systems, modalities, or settings, there also is optimism for incorporating cessation advice and help in multiple venues and vehicles. We need to consider how to make cessation services more attractive to teens, and explore venues outside of the school setting that may present opportunities for intervention. We should look forward to the results of ongoing trials of healthcare provider delivered interventions as well as those with internet based formats. Both of these approaches have the potential for widespread dissemination and reach beyond the traditional school setting.

In thinking about ways to improve the intervention approaches used with adolescent smokers, we also should try to incorporate the "lessons learned" from the adult smoking cessation literature. A major tenet of successful smoking cessation approaches for adults is the acknowledgment that cessation and the maintenance of abstinence are long term processes. There is now substantial evidence from adults that more frequent contact over extended periods of time enhances long term success rates. ${ }^{40}$ However, most of the intervention approaches used with adolescents to date have been relatively brief, without much provision for longer term follow up, or importantly, referrals or hook ups with support systems that are part of the teen's "natural environment". Although smoking cessation interventions with adults that have tried to incorporate social support components (for example, buddy systems, partner training) have met with somewhat limited success, ${ }^{40}{ }^{51}$ they may still be useful avenues to pursue with adolescents. Adolescent smoking is clearly a social phenomenon that is largely tied to peer networks. ${ }^{52}$ Interventions that more specifically address and perhaps capitalise on beneficial peer networks may hold some promise for adolescents.

Relatedly, we also know very little about the relapse process among adolescents. The most basic questions about relapse among adolescent ex-smokers still need to be answered, such as what are the patterns, timing, and predictors of relapse? What interventions work best at preventing relapse, or is the best bet just to work on increasing initial quit rates? We also need to know more about how adolescents interpret failures to quit or to relapse, and how those responses affect future quit attempts and the process of re-engaging youth to quit.
Marketing smoking cessation interventions to youth is also needed. Adolescents need to become comfortable not only with the notion that quitting is important, but also with the concept that help is available and that it is acceptable to seek out help. Adolescents are relatively naive about how and where to get help for quitting. ${ }^{25}$ We also need to understand better the social valence to youth of seeking help for smoking. What social risks do they take in doing so? Besides the possibility of punishment for acknowledging smoking, they may be concerned about negative labels related to being "weak" or "addicted". On the other hand, there also may be other benefits besides quitting that derive from seeking help for cessation (for example, support, incentives). Social marketing approaches for smoking cessation have been useful for adults, and may also serve us well with adolescents. Marketing approaches, though, need to consider the general "background noise" about tobacco and tobacco control in the macro-environment and how these larger tobacco control efforts are interpreted by youth who need to stop smoking. Are we sending mixed messages?

Beyond any one individual intervention approach, though, it is clear that integrated, multilevel approaches are likely to be needed to address adequately the problem of adolescent smoking. Thus, approaches that have broad reaches and are proactive, such as those delivered in the format of brief interventions in healthcare settings, are needed, as well as those that are more limited in reach and may be more appropriate for youth who are primed to quit and responsive to recruitment efforts. Finally, specialised interventions also are likely to be needed for youth who may have multiple, complex problems that interact with their smoking, such as those who have co-morbidities of other substance use, depression, or who have difficult family situations. Although we are not yet at the point where stepped care approaches have been used with adolescents, they may well need to be considered in the future. The time is right for starting to develop the needed infrastructure to support the coordination of referrals among parents, schools, health care providers, and adolescents themselves. The notion of having a menu of services and approaches available for adolescents is likely to have the greatest appeal to the adolescents, their parents, and other stakeholders as well. Important, too, is the need to develop dissemination vehicles to inform the various audiences about cessation services.

We must also consider the possibility that effective intervention programming for adolescents may be relatively expensive, especially when one considers some of the challenges of conducting effective interventions, and the issue of cost effectiveness needs to be considered as well. Although the arguments for intervening with smokers during the adolescent years are compelling, we also need to examine objectively the relative costs and benefits of waiting until the young adult years, when our interventions have greater efficacy. Having an understanding of the total population impact, considering both reach and efficacy, is important. As new and hopefully better interventions and evaluations emerge, we need to start to incorporate broader cost effectiveness and cost utility analyses into the research programmes.

Although the focus of much of this paper has been on outcome, we also need to pay attention to the process of cessation, and to analyses linking process to outcome. In terms of the process of cessation, we need to know more about what are useful intermediate steps or markers to abstinence. For example, are reduction or changes in smoking patterns useful short term goals? How do specific strategies relate to quitting success? Do increases in hypothesised mediators of change (for example, increases in self efficacy, increases in problem solving and coping skills) lead to more successful future quit attempts? Are there optimal time points during adolescence when we should target intervention efforts ( such as transition 
points from schools) or during targets of opportunity or teachable moments (healthcare visits, job or school interviews)? In general, the field would benefit from a clearer understanding of how successful adolescents achieve and maintain abstinence, and what components are linked to an intervention's success. In addition, we have yet to explore systematically the need to tailor interventions to sex or to ethnicity. There is some intriguing preliminary evidence of sex differences in how adolescents respond to interventions and these need to be examined more.

In sum, this paper has posed more questions than it has answered about effective interventions for adolescent smoking cessation, and has left the reader with a promissory note about more and better to come. In the meantime, what can be said about the types of strategies that may be helpful to adolescents as they try to stop smoking? As noted above, strategies need to be available to youth, be perceived as helpful by youth, be appropriate to their developmental stage (which is very heterogeneous), and reflect a diversity in options associated with multiple avenues to success. This diversity of options should also reflect flexibility in implementation that is needed to adapt programmes to specific settings. Little process data currently exist linking specific treatment strategies to outcome, although such data would be useful in further refining interventions for adolescents. The interventions to date represent considerable progress in the total tobacco control landscape from what was available as recently as one decade ago. Although there are no "sure bets", there are promising avenues, which when considered together, may start to make inroads into the complex problem of adolescent smoking cessation.

\section{REFERENCES}

1 US Department of Health and Human Services. Preventing tobacco use among young people. A report of the Surgeon General, 1994. Atlanta, Georgia: Public Health Service, Centers for Disease Control and Prevention, Office on Smoking and Health, 1994. (US Government Printing Office Publication No S/N 017-001-00491-0.)

2 Severson HH, Akers L, Andrews JA, et al. Evaluating two self-help interventions for smokeless tobacco cessation. Addict Behav 2000;25:465-70.

3 Johnston LD, O'Malley PM, Bachman JG. Cigarette smoking among American teens declines sharply in 2001. December 2001. Access date: 24 January 2002. URL: http://www.monitoringthefuture.org

4 Pierce, JP, Gilpin E. How long will today's new adolescent smoker be addicted to cigarettes? Am J Public Health 1996;86:253-6.

5 Stanton WR, McClelland M, Elwood C, et al. Prevalence, reliability and bias of adolescents' reports of smoking and quitting. Addiction 1996:91:1705-14.

6 Stanton WR, Lowe JB, Gillespie, AM. Adolescents' experiences of smoking cessation. Drug Alcohol Depend 1996;43:63-70.

7 Zhu S, Sun J, Billings SC, et al. Predictors of smoking cessation in U.S adolescents. Am J Prev Med 1999;16:202-7.

8 Sussman S, Dent CW, Severson $\mathrm{H}$, et al. Self-initiated quitting among adolescent smokers. Prev Med: an International Devoted to Practice \& Theory 1998;27:A19-28.

9 Chassin L, Presson CC, Pitts SC, et al. The natural history of cigarette smoking from adolescence to adulthood in a midwestern community sample: multiple trajectories and their psychosocial correlates. Health Psychol 2000;19:223-31.

10 US Department of Health and Human Services. Reducing tobacco use: a report of the Surgeon General. Atlanta, Georgia: Centers for Disease Control and Prevention, National Center for Chronic Disease Prevention and Health Promotion, Office on Smoking and Health, 2000 (GPO No. S/N 017-001-00491-0).

11 Colby SM, Tiffany ST, Shiffman S, et al. Are adolescent smokers dependent on nicotine? A review of the evidence. Drug Alcohol Depend 2000;59(suppl 1):S83-95.

12 Kandel DB, Johnson JG, Bird HR, et al. Psychiatric disorders associated with substance use among children and adolescents: findings from the methods for the epidemiology of child and adolescent mental disorders (MECA) study. J Abnormal Child Psych 1997;25:121-32.

13 Prokhorov AV, Pallonen UE, Fava JL, et al. Measuring nicotine dependence among high-risk adolescent smokers. Addict Behav 1996;21:117-27.

14 Smith TA, House RF Jr, Croghan IT, et al. Nicotine patch therapy in adolescent smokers. Pediatrics 1996;98(4 Pt 1):659-67.

15 Eissenberg T, Balster RL. Initial tobacco use episodes in children and adolescents: current knowledge, future directions. Drug Alcohol Depend 2000;59(suppl 1):S41-60.
16 DiFranza JR, Rigotti NA, McNeill AD, et al Initial symptoms of nicotine dependence in adolescents. Tobacco Control 2000;9:313-9.

17 Shadel WG, Shiffman S, Niaura R, et al. Current models of nicotine dependence: what is known and what is needed to advance understanding of tobacco etiology among youth. Drug Alcohol Depend 2000;59:S9-21.

18 Sussman S, Dent CW, Nezami E, et al. Reasons for quitting and smoking temptation among adolescent smokers: gender differences. Subst Use Misuse 1998;33:2703-20.

19 Centers for Disease Control. Reasons for tobacco use and symptoms of nicotine withdrawal among adolescent and young adult tobacco users - United States, 1993. MMWR Morb Mortal Wkly Rep 1994;43:745-50

20 Engels RC, Knibbe, RA, De Vries H, et al. Antecendents of smoking cessation among adolescents: who is motivated to change. Prev Med 1998:27:348-57

21 Pallonen UE, Prochaska JO, Velicer WF, et al. Stages of acquisition and cessation for adolescent smoking: an empirical integration. Addict Behav 1998;23:303-24

22 Stanton WR. DSM-III-R tobacco dependence and quitting during late adolescence. Addict Behav 1995:20:595-603.

23 Stone SL, Kristeller JL. Attitudes of adolescents toward smoking cessation. Am J Prev Med 1992;8:221-5.

24 Fisher KJ, Stanton WR, Lowe JB. Healthy behaviors, lifestyle, and reasons for quitting smoking among out-of-school youth. J Adolesc Health 1999:25:290-7.

25 Balch GI. Exploring perceptions of smoking cessation among high school smokers: input and feedback from focus groups. Prev Med: an International Devoted to Practice \& Theory 1998;27(5):A55-63

26 Sussman S. Effects of sixty six adolescent tobacco use cessation trials and seventeen prospective studies of self-initiated quitting. Tobacco Induced Diseases 2002;1:35-81.

27 Sussman S, Lichtman K, Ritt A. Effects of 34 adolescent tobacco use cessation and prevention trials on regular users of tobacco products. Subst Use Misuse 1999;34:1469-503.

28 Dino G, Horn K, Goldcamp J, et al. A 2-year efficacy study of Not On Tobacco in Florida: an overview of program successes in changing teen smoking behavior. Prev Med 2001;33:600-5

29 Lampkin L. "Meet it head on" evaluation report [technical report] Chicago, Illinois: American Medical Association, 1998.

30 Colby SM, Monti PM, Barnett NP, et al. 1998. Brief motivational interviewing in a hospital setting for adolescent smoking: a preliminary study. J Consult Clin Psychol 1998;66:574-8.

31 Woodruff SI, Edwards CC, Conway TL, et al. Pilot test of an internet virtual world chat room for rural teen smokers. J Adolesc Health 2001;29:239-43

32 Perry CL, Killen J, Slinkard LA, et al. Peer teaching and smoking prevention among junior high students. Adolescence 1980;15:277-81.

33 Coleman-Wallace D, Lee JW, Montgomery S, et al. Evaluation of developmentally appropriate programs for adolescent tobacco cessation. J School Health 1999;69:314-9.

34 Quinlan KB, McCaul KD. Matched and mismatched interventions with young adult smokers: testing a stage theory. Health Psychol $2000 ; 19,165-71$

35 Mermelstein RJ, Karnatz T, Reichmann S. Smoking. In: Wilson PH, ed. Principles and practice of relapse prevention. New York: The Guilford Press, 1992.

36 Sussman S, Dent CW, Lichtman KL. Project EX: outcomes of a teen smoking cessation program. Addict Behav 2001;26:425-38.

37 Turner LR, Veldhuis CB, Mermelstein RJ. Predictors of drop-out in a school-based adolescent smoking cessation program. Presented at Society for Behavioral Medicine, Washington, DC, 2-6 April 2002

38 Dino GA, Horn KA, Goldcamp J, et al. Statewide demonstration of Not On Tobacco: a gender-sensitive teen smoking cessation program. Journal of School Nursing 2001;17:90-7.

39 Hurt RD, Croghan GA, Beede SD, et al. Nicotine patch therapy in 101 adolescent smokers: efficacy, withdrawal symptom relief, and carbon monoxide and plasma cotinine levels. Arch Pediatr Adolesc Med 2000;154:31-7.

40 Fiore MC, Bailey WC, Cohen SJ, et al. A clinical practice guideline for treating tobacco use and dependence - A US Public Health Service report. JAMA 2000;283:3244-54

41 Glasgow RE, Strycker LA, Eakin EG, et al. Concern about weight gain associated with quitting smoking: prevalence and association with outcome in a sample of young female smokers. J Consult Clin Psychol 1999:67:1009-11.

42 Hollis JF, Vogt TM, Stevens V, et al. The tobacco reduction and cancer control (TRACC) program: team approaches to counseling in medical and dental settings. In National Cancer Institute, Tobacco and the clinician: interventions for medical and dental practice. Smoking and Tobacco Control Monograph No. 5. USDHHS, 1994: 143-85. (NIH Publication No. 94-3693.

43 Hollis J, Polen M, Whitlock E, et al. Efficacy of a brief tobacco prevention and cessation program for teens seen in routine medical care (TEEN REACH). Paper presented at the Annual Meeting of the Society for Behavioral Medicine, Washington, DC, April 2002.

44 Cheh JA, Ribisl KM, Wildemuth BM. An assessment of the quality and usability of smoking cessation information on the internet. Health Promotion Practice (in press)

45 Bauman KE, Ennett ST, Foshee VA, et al. Influence of a family-directed program on adolescent cigarette and alcohol cessation. Prev $\mathrm{Sci}$ 2000; $1: 227-37$ 
46 Mermelstein R. Explanations of ethnic and gender differences in youth smoking: a multi-site, qualitative investigation. Nicotine Tob Res 1999; 1:S91-8.

47 Moolchan E Mermelstein R. Tobacco research among teenagers: the bumpy road of ethical challenges. J Adolesc Health 2002;30:409-17

48 Brooks-Gunn, J. Why do adolescents have difficulty adhering to health regimes? In: Krasnegor NA, Epstein LH, eds. Developmental aspects of health compliance behavior. Hillsdale, New Jersey: Lawrence Erlbaum Associates, Inc, 1993
49 McMahon RJ. Child and adolescent psychopathology as risk factors for subsequent tobacco use. Nicotine Tob Res 1999; 1:S45-50.

50 Mermelstein R, Colby SM, Patten CA, et al. Methodological issues in measuring treatment outcome in adolescent smoking cessation studies. Nicotine Tob Res. (in press).

51 Cohen S, Lichtenstein E, Kingsolver K, et al. Social support interventions for smoking cessation. In: Gottlieb BH, ed. Marshaling social support: formats, processes, and effects. Thousand Oaks, California: Sage Publications, 1988 .

52 Kobus, K. Peers and adolescent smoking. Addiction (in press). 